Kragujevac Journal of Mathematics

Volume 46(1) (2022), Pages 85-104.

\title{
EXISTENCE AND STABILITY ANALYSIS OF SEQUENTIAL COUPLED SYSTEM OF HADAMARD-TYPE FRACTIONAL DIFFERENTIAL EQUATIONS
}

\author{
AKBAR ZADA ${ }^{1}$ AND MOHAMMAD YAR ${ }^{1}$
}

\begin{abstract}
In this paper we study existence, uniqueness and Hyers-Ulam stability for a sequential coupled system consisting of fractional differential equations of Hadamard type, subject to nonlocal Hadamard fractional integral boundary conditions. The existence of solutions is derived from Leray-Schauder's alternative, whereas the uniqueness of solution is established by Banach contraction principle. An example is also presented which illustrate our results.
\end{abstract}

\section{INTRODUCTION}

In this paper, we study the sequential coupled system of Hadamard fractional differential equations with nonlocal Hadamard fractional integral boundary conditions of the following form:

$$
\left\{\begin{array}{l}
\left(D^{q}+k D^{q-1}\right) u(t)=f(t, u(t), v(t)), \quad k>0,1<q \leq 2, t \in(1, e), \\
\left(D^{p}+k D^{p-1}\right) v(t)=g(t, u(t), v(t)), \quad k>0,1<p \leq 2, t \in(1, e), \\
u(1)=0, \quad \sum_{i=1}^{m} \lambda_{i} I^{\alpha_{i}} u\left(\eta_{i}\right)=\sum_{j=1}^{n} \mu_{j}\left(I^{\beta_{j}} u(e)-I^{\beta_{j}} u\left(\xi_{j}\right)\right), \\
v(1)=0, \quad \sum_{i=1}^{m} \rho_{i} I^{\gamma_{i}} v\left(\theta_{i}\right)=\sum_{j=1}^{n} \kappa_{j}\left(I^{\delta_{j}} v(e)-I^{\delta_{j}} v\left(\zeta_{j}\right)\right)
\end{array}\right.
$$

where $D^{(\cdot)}$ denotes the Hadamard fractional derivative of order $p$ and $q, f, g:[1, e] \times$ $\mathbb{R}^{2} \rightarrow \mathbb{R}$ are continuous functions and $\eta_{i}, \theta_{i}, \xi_{j}, \zeta_{j} \in(1, e)$ and $\lambda_{i}, \rho_{i}, \mu_{j}, \kappa_{j} \in \mathbb{R}$,

Key words and phrases. Hadamard fractional derivative, sequential coupled system, fixed point theorem, Hyers-Ulam stability.

2010 Mathematics Subject Classification. Primary: 26A33. Secondary: 34A08, 35B40.

DOI 10.46793/KgJMat2201.085Z

Received: April 07, 2019.

Accepted: September 01, 2019. 
$i=1,2, \ldots, m, j=1,2, \ldots, n$, and $I^{(\phi)}$ is the Hadamard fractional integral of order $\phi>0, \phi=\alpha_{i}, \gamma_{i}, \beta_{j}, \delta_{j}, i=1,2, \ldots, m, j=1,2, \ldots, n$.

Fractional calculus is the field of mathematical analysis, which deals with the investigation and applications of integrals and derivatives of an arbitrary order. Fractional differential equations (FDEs) have played a significant role in many engineering and scientific disciplines e.g. as the mathematical modeling of systems and processes in the fields of physics, chemistry, aerodynamics, electrodynamics of complex medium, polymer rheology, bode's analysis of feedback amplifiers, capacitor theory, electrical circuits, electron analytical chemistry, biology, control theory, fitting of experimental data and so forth $[5,14,15]$. FDEs also serve as an excellent tool for the description of hereditary properties of various materials and processes [18].

The theory of fractional order differential equations, involving different kinds of boundary conditions has been a field of interest in pure and applied sciences. Nonlocal conditions are used to describe certain features of applied mathematics and physics such as blood flow problems, chemical engineering, thermo-elasticity, underground water flow, population dynamics and so on $[1,6,17]$.

In the classical text [19], it has been mentioned that Hadamard in 1892 [8] suggested a concept of fractional integro-differentiation in terms of the fractional power of the type $\left(x \frac{d}{d x}\right)^{p}$ in contrast to its Riemann-Liouville counterpart of the form $\left(\frac{d}{d x}\right)^{p}$. The kind of derivative, introduced by Hadamard contains logarithmic function of arbitrary exponent in the kernel of the integral appearing in its definition. Hadamard's construction is invariant in relation to dilation and is well suited to the problems containing half axes.

Coupled systems of FDEs have also been investigated by many authors. Such systems appear naturally in many real world situations. Some recent results on the topic can be found in a series of papers $[2,3,9,12,20,25]$.

Another aspect of FDEs which has very recently got attentions from the researchers is the Ulam type stability analysis of the aforesaid equations. The mentioned stability was first pointed out by Ulam [26] in 1940, which was further explained by Hyers [10], over Banach space. Latter on, many researchers done valuable work on the same task and interesting results were formed for linear and nonlinear integral and differential equations, for detail see $[4,24,27,28,30-32,35]$. This stability analysis is very useful in many applications, such as numerical analysis, optimization, etc., where finding the exact solution is quite difficult. For detailed study of Ulam-type stability with different approaches, we recommend papers [13,21-24, 29, 33, 34,36-39].

In addition, the inspiration of this paper comes from the following two problems $[11,16]$. In [16], Thiramanus et al. investigated the existence and uniqueness of solutions for a fractional boundary value problem involving Hadamard-type fractional 
differential equations and nonlocal fractional integral boundary conditions:

$$
\left\{\begin{array}{l}
D^{q} x(t)=f(t, x(t)), \quad 1<q \leq 2, t \in(1, e), \\
x(1)=0 \\
\sum_{i=1}^{m} \lambda_{i} I^{\alpha_{i}} x\left(\eta_{i}\right)=\sum_{j=1}^{n} \mu_{j}\left(I^{\beta_{j}} x(e)-I^{\beta_{j}} x\left(\xi_{j}\right)\right)
\end{array}\right.
$$

where $D^{q}$ denotes the Hadamard fractional derivative of order $\mathrm{q}, f:[1, e] \times \mathbb{R} \rightarrow \mathbb{R}$ is a continuous function, $\eta_{i}, \xi_{j} \in(1, e), \lambda_{i}, \mu_{j} \in \mathbb{R}$ for all $i=1,2, \ldots, m, j=1,2, \cdots, n$, $\eta_{1}<\eta_{2}<\cdots<\eta_{m}, \xi_{1}<\xi_{2}<\cdots<\xi_{n}$ and $I \phi$ is the Hadamard fractional integral of order $\phi>0, \phi=\alpha_{i}, \beta_{j}, i=1,2, \ldots, m, j=1,2, \ldots, n$.

In [11], the authors discussed existence results for Hadamard type fractional functional integro-differential equations with integral boundary conditions:

$$
\left\{\begin{array}{l}
D^{q} y(t)=f\left(t, y(t),\left(T_{1} y\right)(t),\left(T_{2} y\right)(t)\right), \quad 1<q \leq 2, t \in(1, e) \\
y(1)=0 \\
\sum_{i=1}^{m} \lambda_{i} I^{\alpha_{i}} y\left(\eta_{i}\right)=\sum_{j=1}^{n} \mu_{j}\left(I^{\beta_{j}} x(e)-I^{\beta_{j}} x\left(\xi_{j}\right)\right) .
\end{array}\right.
$$

We show the existence of solutions for problem (1.1) by applying Leray-Schauder alternative criterion while uniqueness of solutions for (1.1) relies on Banach contraction mapping principle.

The rest of the paper is organized as follows: In Section 2, we recall some preliminary concepts which we need in the sequel. Section 3 contains the main results for problem (1.1). In Section 4, we present the Hyers-Ulam stability for problem (1.1).

\section{Preliminaries and Background Materials}

In this section, we introduce some notations and definitions of fractional calculus and present preliminary results needed in our proofs, and then we prove an auxiliary lemma for the linear modification of problem (1.1).

Definition 2.1. The Hadamard derivative of fractional order $q$ for a function $f \in$ $C^{n}[1, \infty)$, is defined as

$$
D^{q} f(t)=\frac{1}{\Gamma(n-q)}\left(t \frac{d}{d t}\right)^{n} \int_{0}^{t}\left(\log \frac{t}{s}\right)^{n-q-1} \frac{f(s)}{s} d s, \quad n-1<q<n,
$$

where $n=[q]+1,[q]$ denotes the integer part of $q$ and $\log (\cdot)=\log _{e}(\cdot)$ provided that integral exists.

Definition 2.2. The Hadamard fractional integral of order $q$ for a function $f$ : $[1, \infty) \rightarrow \mathbb{R}$ is defined by

$$
I^{q} f(t)=\frac{1}{\Gamma(q)} \int_{1}^{t}\left(\log \frac{t}{s}\right)^{q-1} \frac{f(s)}{s} d s, \quad q>0
$$


provided that integral exists, and $\Gamma(\cdot)$ is the Gamma function defined by

$$
\Gamma(q)=\int_{0}^{\infty} e^{-s} t^{q-1} d s, \quad q>0 .
$$

Lemma 2.1. Let $\phi, \psi$ are continuous functions from $[1, e]$ to $\mathbb{R}$. Then the solution for the linear system of sequential fractional differential equations:

$$
\left\{\begin{array}{l}
\left(D^{q}+k D^{q-1}\right) u(t)=\phi(t), \quad k>0,1<q \leq 2, t \in(1, e), \\
\left(D^{p}+k D^{p-1}\right) v(t)=\psi(t), \quad 1<p \leq 2, t \in(1, e), \\
u(1)=0, \quad \sum_{i=1}^{m} \lambda_{i} I^{\alpha_{i}} u\left(\eta_{i}\right)=\sum_{j=1}^{n} \mu_{j}\left(I^{\beta_{j}} u(e)-I^{\beta_{j}} u\left(\xi_{j}\right)\right), \\
v(1)=0, \quad \sum_{i=1}^{m} \rho_{i} I^{\gamma_{i}} v\left(\theta_{i}\right)=\sum_{j=1}^{n} \kappa_{j}\left(I^{\delta_{j}} v(e)-I^{\delta_{j}} v\left(\zeta_{j}\right)\right),
\end{array}\right.
$$

is

$$
\begin{aligned}
u(t)= & \frac{1}{A_{1}}\left(t^{-k} \int_{1}^{t} s^{k-1}(\log s)^{q-2} d s\right)\left[\sum_{i=1}^{m} \frac{\lambda_{i}}{\Gamma\left(\alpha_{i}\right) \Gamma(q-1)}\right. \\
& \times \int_{1}^{\eta_{i}}\left(\log \frac{\eta_{i}}{s}\right)^{\alpha_{i}-1} s^{-k-1}\left(\int_{1}^{s} r^{k-1}\left(\int_{1}^{r}\left(\log \frac{r}{m}\right)^{q-2} \frac{\phi(m)}{m} d m\right) d r\right) d s \\
& +\sum_{j=1}^{n} \frac{\mu_{j}}{\Gamma\left(\beta_{j}\right) \Gamma(q-1)} \int_{1}^{\xi_{j}}\left(\log \frac{\xi_{j}}{s}\right)^{\beta_{j}-1} s^{-k-1} \\
& \times\left(\int_{1}^{s} r^{k-1}\left(\int_{1}^{r}\left(\log \frac{r}{m}\right)^{q-2} \frac{\phi(m)}{m} d m\right) d r\right) d s \\
& -\frac{e^{-k}}{\Gamma(q-1)} \sum_{j=1}^{n} \frac{\mu_{j}}{\Gamma\left(\beta_{j}\right)} \int_{1}^{e}\left(\log \frac{e}{s}\right)^{\beta_{j}-1} s^{-1} \\
& \left.\times\left(\int_{1}^{e} r^{k-1}\left(\int_{1}^{r}\left(\log \frac{r}{m}\right)^{q-2} \frac{\phi(m)}{m} d m\right) d r\right) d s\right] \\
& +t^{-k} \int_{1}^{t} s^{k-1} I^{q-1} \phi(s) d s
\end{aligned}
$$

and

$$
\begin{aligned}
v(t)= & \frac{1}{A_{2}}\left(t^{-k} \int_{1}^{t} s^{k-1}(\log s)^{p-2} d s\right)\left[\sum_{i=1}^{m} \frac{\rho_{i}}{\Gamma\left(\gamma_{i}\right) \Gamma(p-1)}\right. \\
& \times \int_{1}^{\theta_{i}}\left(\log \frac{\theta_{i}}{s}\right)^{\gamma_{i}-1} s^{-k-1}\left(\int_{1}^{s} r^{k-1}\left(\int_{1}^{r}\left(\log \frac{r}{m}\right)^{p-2} \frac{\psi(m)}{m} d m\right) d r\right) d s \\
& +\sum_{j=1}^{n} \frac{\kappa_{j}}{\Gamma\left(\delta_{j}\right) \Gamma(p-1)} \int_{1}^{\zeta_{j}}\left(\log \frac{\zeta_{j}}{s}\right)^{\delta_{j}-1} s^{-k-1} \\
& \times\left(\int_{1}^{s} r^{k-1}\left(\int_{1}^{r}\left(\log \frac{r}{m}\right)^{p-2} \frac{\psi(m)}{m} d m\right) d r\right) d s
\end{aligned}
$$




$$
\begin{aligned}
& -\frac{e^{-k}}{\Gamma(p-1)} \sum_{j=1}^{n} \frac{\kappa_{j}}{\Gamma\left(\delta_{j}\right)} \int_{1}^{e}\left(\log \frac{e}{s}\right)^{\delta_{j}-1} s^{-1} \\
& \left.\times\left(\int_{1}^{e} r^{k-1}\left(\int_{1}^{r}\left(\log \frac{r}{m}\right)^{p-2} \frac{\psi(m)}{m} d m\right) d r\right) d s\right] \\
& +t^{-k} \int_{1}^{t} s^{k-1} I^{p-1} \psi(s) d s
\end{aligned}
$$

where

$$
\begin{aligned}
A_{1}= & \sum_{j=1}^{n} \frac{e^{-k} \mu_{j}}{\Gamma\left(\beta_{j}\right)} \int_{1}^{e}\left(\log \frac{e}{s}\right)^{\beta_{j}-1} s^{-1}\left(\int_{1}^{e} r^{k-1}(\log r)^{q-2} d r\right) d s \\
& -\sum_{j=1}^{n} \frac{\mu_{j}}{\Gamma\left(\beta_{j}\right)} \int_{1}^{\xi_{j}}\left(\log \frac{\xi_{j}}{s}\right)^{\beta_{j}-1} s^{-k-1}\left(\int_{1}^{r} r^{k-1}(\log r)^{q-2} d r\right) d s \\
& -\sum_{i=1}^{m} \frac{\lambda_{i}}{\Gamma\left(\alpha_{i}\right)} \int_{1}^{\eta_{i}}\left(\log \frac{\eta_{i}}{s}\right)^{\alpha_{i}-1} s^{-k-1}\left(\int_{1}^{r} r^{k-1}(\log r)^{q-2} d r\right) d s
\end{aligned}
$$

and

$$
\begin{aligned}
A_{2}= & \sum_{j=1}^{n} \frac{e^{-k} \kappa_{j}}{\Gamma\left(\delta_{j}\right)} \int_{1}^{e}\left(\log \frac{e}{s}\right)^{\delta_{j}-1} s^{-1}\left(\int_{1}^{e} r^{k-1}(\log r)^{p-2} d r\right) d s \\
& -\sum_{j=1}^{n} \frac{\kappa_{j}}{\Gamma\left(\delta_{j}\right)} \int_{1}^{\zeta_{j}}\left(\log \frac{\zeta_{j}}{s}\right)^{\delta_{j}-1} s^{-k-1}\left(\int_{1}^{r} r^{k-1}(\log r)^{p-2} d r\right) d s \\
& -\sum_{i=1}^{m} \frac{\rho_{i}}{\Gamma\left(\gamma_{i}\right)} \int_{1}^{\theta_{i}}\left(\log \frac{\theta_{i}}{s}\right)^{\gamma_{i}-1} s^{-k-1}\left(\int_{1}^{r} r^{k-1}(\log r)^{p-2} d r\right) d s .
\end{aligned}
$$

Proof. As argued in [14], the general solution of the system (2.1) can be written as

$$
u(t)=c_{0} t^{-k}+c_{1} t^{-k} \int_{1}^{t} s^{k-1}(\log s)^{q-2} d s+t^{-k} \int_{1}^{t} s^{k-1} I^{q-1} \phi(t) d s
$$

and

$$
v(t)=d_{0} t^{-k}+d_{1} t^{-k} \int_{1}^{t} s^{k-1}(\log s)^{p-2} d s+t^{-k} \int_{1}^{t} s^{k-1} I^{p-1} \psi(t) d s,
$$

where $c_{i}, d_{i}, i=0,1$, are unknown arbitrary constants. The conditions $u(1)=0$ and $v(1)=0$ in (2.6) and (2.7) imply that $c_{0}=0$ and $d_{0}=0$, which leads to

$$
u(t)=c_{1} t^{-k} \int_{1}^{t} s^{k-1}(\log s)^{q-2} d s+t^{-k} \int_{1}^{t} s^{k-1} I^{q-1} \phi(t) d s
$$

and

$$
v(t)=d_{1} t^{-k} \int_{1}^{t} s^{k-1}(\log s)^{p-2} d s+t^{-k} \int_{1}^{t} s^{k-1} I^{p-1} \psi(t) d s .
$$


Now, using the coupled integral boundary conditions given by (1.1), in (2.8) and (2.9), we obtain

$$
c_{1}=\frac{J_{1}}{A_{1}}, \quad d_{1}=\frac{J_{2}}{A_{2}},
$$

where $A_{1}$ and $A_{2}$ are respectively given by (2.4) and (2.5), and

$$
\begin{aligned}
J_{1}= & \sum_{i=1}^{m} \frac{\lambda_{i}}{\Gamma\left(\alpha_{i}\right) \Gamma(q-1)} \int_{1}^{\eta_{i}}\left(\log \frac{\eta_{i}}{s}\right)^{\alpha_{i}-1} s^{-k-1} \\
& \times\left(\int_{1}^{s} r^{k-1}\left(\int_{1}^{r}\left(\log \frac{r}{m}\right)^{q-2} \frac{\phi(m)}{m} d m\right) d r\right) d s \\
& +\sum_{j=1}^{n} \frac{\mu_{j}}{\Gamma\left(\beta_{j}\right) \Gamma(q-1)} \int_{1}^{\xi_{j}}\left(\log \frac{\xi_{j}}{s}\right)^{\beta_{j}-1} s^{-k-1} \\
& \times\left(\int_{1}^{s} r^{k-1}\left(\int_{1}^{r}\left(\log \frac{r}{m}\right)^{q-2} \frac{\phi(m)}{m} d m\right) d r\right) d s \\
& -\sum_{j=1}^{n} \frac{e^{-k} \mu_{j}}{\Gamma\left(\beta_{j}\right)} \int_{1}^{e}\left(\log \frac{e}{s}\right)^{\beta_{j}-1} s^{-1}\left(\int_{1}^{e} r^{k-1} I^{q-1} \phi(r) d r\right) d s \\
J_{2}= & \sum_{i=1}^{m} \frac{\rho_{i}}{\Gamma\left(\gamma_{i}\right) \Gamma(p-1)} \int_{1}^{\theta_{i}}\left(\log \frac{\theta_{i}}{s}\right)^{\gamma_{i}-1} s^{-k-1} \\
& \times\left(\int_{1}^{s} r^{k-1}\left(\int_{1}^{r}\left(\log \frac{r}{m}\right)^{p-2} \frac{\psi(m)}{m} d m\right) d r\right) d s \\
& +\sum_{j=1}^{n} \frac{\kappa_{j}}{\Gamma\left(\delta_{j}\right) \Gamma(p-1)} \int_{1}^{\zeta_{j}}\left(\log \frac{\zeta_{j}}{s}\right)^{\delta_{j}-1} s^{-k-1} \\
& \times\left(\int_{1}^{s} r^{k-1}\left(\int_{1}^{r}\left(\log \frac{r}{m}\right)^{p-2} \frac{\psi(m)}{m} d m\right) d r\right) d s \\
& -\sum_{j=1}^{n} \frac{e^{-k} \kappa_{j}}{\Gamma\left(\delta_{j}\right)} \int_{1}^{e}\left(\log \frac{e}{s}\right)^{\delta_{j}-1} s^{-1}\left(\int_{1}^{e} r^{k-1} I^{p-1} \psi(r) d r\right) d s .
\end{aligned}
$$

Substituting the values of $c_{1}$ and $c_{2}$ in (2.8) and (2.9) we obtained the solutions (2.2) and (2.3).

\section{Main Results}

Let $\mathcal{C}=C([1, e], \mathbb{R})$ denotes the Banach space of all continuous functions from $[1, e]$ to $\mathbb{R}$. Let us introduce the space $X=\left\{u(t): u(t) \in C^{1}([1, e])\right\}$ endowed with the norm $\|u\|=\sup \{|u(t)|: t \in[1, e]\}$. Obviously, $(X,\|\cdot\|)$ is a Banach space. Also let $Y=\left\{v(t): v(t) \in C^{1}([1, e])\right\}$ be endowed with the norm $\|v\|=\sup \{|v(t)|: t \in[1, e]\}$. Obviously the product space $(X \times Y,\|(u, v)\|)$ is a Banach space with norm $\|(u, v)\|=$ 
$\|u\|+\|v\|$. In view of Lemma 2.1, we define an operator $\mathcal{T}: X \times Y \rightarrow X \times Y$ by

$$
\mathcal{T}(u, v)(t)=\left(\begin{array}{c}
\mathcal{T}_{1}(u, v)(t) \\
\mathcal{T}_{2}(u, v)(t)
\end{array}\right)
$$

where

$$
\begin{aligned}
\mathcal{T}_{1}(u, v)(t)= & \frac{1}{A_{1}}\left(t^{-k} \int_{1}^{t} s^{k-1}(\log s)^{q-2} d s\right) \\
& {\left[\sum_{i=1}^{m} \frac{\lambda_{i}}{\Gamma\left(\alpha_{i}\right)} \int_{1}^{\eta_{i}}\left(\log \frac{\eta_{i}}{s}\right)^{\alpha_{i}-1} s^{-k-1}\left(\int_{1}^{s} r^{k-1}\left(I^{q-1} f(r, u(r), v(r))\right) d r\right) d s\right.} \\
& +\sum_{j=1}^{n} \frac{\mu_{j}}{\Gamma\left(\beta_{j}\right)} \int_{1}^{\xi_{j}}\left(\log \frac{\xi_{j}}{s}\right)^{\beta_{j}-1} s^{-k-1}\left(\int_{1}^{s} r^{k-1}\left(I^{q-1} f(r, u(r), v(r))\right) d r\right) d s \\
& \left.-\sum_{j=1}^{n} \frac{e^{-k} \mu_{j}}{\Gamma\left(\beta_{j}\right)} \int_{1}^{e}\left(\log \frac{e}{s}\right)^{\beta_{j}-1} s^{-1}\left(\int_{1}^{e} r^{k-1} I^{q-1} f(r, u(r), v(r)) d r\right) d s\right] \\
& +t^{-k} \int_{1}^{t} s^{k-1} I^{q-1} f(s, u(s), v(s)) d s
\end{aligned}
$$

and

$$
\begin{aligned}
\mathcal{T}_{2}(u, v)(t)= & \frac{1}{A_{2}}\left(t^{-k} \int_{1}^{t} s^{k-1}(\log s)^{p-2} d s\right) \\
& {\left[\sum_{i=1}^{m} \frac{\rho_{i}}{\Gamma\left(\gamma_{i}\right)} \int_{1}^{\theta_{i}}\left(\log \frac{\theta_{i}}{s}\right)^{\gamma_{i}-1} s^{-k-1}\left(\int_{1}^{s} r^{k-1}\left(I^{p-1} g(r, u(r), v(r))\right) d r\right) d s\right.} \\
& +\sum_{j=1}^{n} \frac{\kappa_{j}}{\Gamma\left(\delta_{j}\right)} \int_{1}^{\zeta_{j}}\left(\log \frac{\zeta_{j}}{s}\right)^{\delta_{j}-1} s^{-k-1}\left(\int_{1}^{s} r^{k-1}\left(I^{p-1} g(r, u(r), v(r))\right) d r\right) d s \\
& \left.-\sum_{j=1}^{n} \frac{e^{-k} \kappa_{j}}{\Gamma\left(\delta_{j}\right)} \int_{1}^{e}\left(\log \frac{e}{s}\right)^{\delta_{j}-1} s^{-1}\left(\int_{1}^{e} r^{k-1} I^{p-1} g(r, u(r), v(r)) d r\right) d s\right] \\
& +t^{-k} \int_{1}^{t} s^{k-1} I^{p-1} g(s, u(s), v(s)) d s .
\end{aligned}
$$

For the sake of convenience, we set

$$
\begin{aligned}
M_{1}= & \frac{1}{\left|A_{1}\right|}\left(\sum_{i=1}^{m} \frac{\left|\lambda_{i}\right|\left(\log \eta_{i}\right)^{\alpha_{i}+q}}{(q-1) \Gamma(q+1) \Gamma\left(\alpha_{i}+1\right)}+\sum_{j=1}^{n} \frac{\left|\mu_{j}\right|\left(\log \xi_{j}\right)^{\beta_{j}+q}}{(q-1) \Gamma(q+1) \Gamma\left(\beta_{j}+1\right)}\right) \\
& +\sum_{j=1}^{n} \frac{\left|\mu_{j}\right|}{(q-1) \Gamma(q+1) \Gamma\left(\beta_{j}+1\right)}, \\
M_{2}= & \frac{1}{\left|A_{2}\right|}\left(\sum_{i=1}^{m} \frac{\left|\rho_{i}\right|\left(\log \theta_{i}\right)^{\gamma_{i}+p}}{(p-1) \Gamma(p+1) \Gamma\left(\gamma_{i}+1\right)}+\sum_{j=1}^{n} \frac{\left|\kappa_{j}\right|\left(\log \zeta_{j}\right)^{\delta_{j}+p}}{(p-1) \Gamma(p+1) \Gamma\left(\delta_{j}+1\right)}\right)
\end{aligned}
$$




$$
+\sum_{j=1}^{n} \frac{\left|\kappa_{j}\right|}{(p-1) \Gamma(p+1) \Gamma\left(\delta_{j}+1\right)}
$$

and

$$
M_{0}=\min \left\{1-\left(M_{1} k_{1}+M_{2} \lambda_{1}\right), 1-\left(M_{1} k_{2}+M_{2} \lambda_{2}\right\}, \quad k_{i}, \lambda_{i} \leq 0, i=1,2 .\right.
$$

The first result is concerned with the existence and uniqueness of solution for the problem (1.1) and is based on Banach contraction mapping principle.

Theorem 3.1. Assume that $f, g:[1, e] \times \mathbb{R}^{\not} \rightarrow \mathbb{R}$ are continuous functions and there exist constants $m_{i}, n_{i}, i=1,2$ such that for all $t \in[1, e]$ and $u_{i}, v_{i} \in \mathbb{R}, i=1,2$, such that

$$
\left|f\left(t, u_{2}, v_{2}\right)-f\left(t, u_{1}, v_{1}\right)\right| \leq m_{1}\left|u_{2}-u_{1}\right|+m_{2}\left|v_{2}-v_{1}\right|
$$

and

$$
\left|g\left(t, u_{2}, v_{2}\right)-g\left(t, u_{1}, v_{1}\right)\right| \leq n_{1}\left|u_{2}-u_{1}\right|+n_{2}\left|v_{2}-v_{1}\right| .
$$

In addition, assume that

$$
M_{1}\left(m_{1}+m_{2}\right)+M_{2}\left(\left(n_{1}+n_{2}\right)<1,\right.
$$

where $M_{i}, i=1,2$, are given by (3.3) and (3.4). Then the boundary value problem (1.1) has a unique solution.

Proof. Define $\sup _{t \in[1, e]} f(t, 0,0)=N_{1}<\infty$ and $\sup _{t \in[1, e]} g(t, 0,0)=N_{2}<\infty$ such that

$$
r>\frac{M_{1} N_{1}+M_{2} N_{2}}{1-M_{1}\left(m_{1}+m_{2}\right)+M_{2}\left(n_{1}+n_{2}\right)} .
$$

We show that $\mathcal{T} \mathbf{B}_{\mathbf{r}} \subset \mathbf{B}_{\mathbf{r}}$, where $\mathbf{B}_{\mathbf{r}}=\{(u, v) \in X \times Y:\|(u, v)\|<r\}$. For $(u, v) \in \mathbf{B}_{\mathbf{r}}$, we have

$$
\begin{aligned}
\left|\mathcal{T}_{1}(u, v)(t)\right|= & \sup _{t \in[1, e]}\left\{\frac { 1 } { A _ { 1 } } ( t ^ { - k } \int _ { 1 } ^ { t } s ^ { k - 1 } ( \operatorname { l o g } s ) ^ { q - 2 } d s ) \left[\sum_{i=1}^{m} \frac{\lambda_{i}}{\Gamma\left(\alpha_{i}\right) \Gamma(q-1)}\right.\right. \\
& \times \int_{1}^{\eta_{i}}\left(\log \frac{\eta_{i}}{s}\right)^{\alpha_{i}-1} s^{-k-1}\left(\int _ { 1 } ^ { s } r ^ { k - 1 } \left(\int_{1}^{r}\left(\log \frac{r}{m}\right)^{q-2}\right.\right. \\
& \left.\left.\times \frac{f(m, u(m), v(m))}{m} d m\right) d r\right) d s+\sum_{j=1}^{n} \frac{\mu_{j}}{\Gamma\left(\beta_{j}\right) \Gamma(q-1)} \\
& \times \int_{1}^{\xi_{j}}\left(\log \frac{\xi_{j}}{s}\right)^{\beta_{j}-1} s^{-k-1}\left(\int _ { 1 } ^ { s } r ^ { k - 1 } \left(\int_{1}^{r}\left(\log \frac{r}{m}\right)^{q-2}\right.\right. \\
& \left.\left.\frac{f(m, u(m), v(m))}{m} d m\right) d r\right) d s-\sum_{j=1}^{n} \frac{e^{-k} \mu_{j}}{\Gamma\left(\beta_{j}\right) \Gamma(q-1)} \int_{1}^{e}\left(\log \frac{e}{s}\right)^{\beta_{j}-1} s^{-1} \\
& \left.\times\left(\int_{1}^{e} r^{k-1}\left(\int_{1}^{r}\left(\log \frac{r}{m}\right)^{q-2} \frac{f(m, u(m), v(m))}{m} d m\right) d r\right) d s\right]
\end{aligned}
$$




$$
\begin{aligned}
& \left.+\frac{t^{-k}}{\Gamma(q-1)} \int_{1}^{t} s^{k-1}\left(\int_{1}^{s}\left(\log \frac{s}{r}\right)^{q-2} \frac{f(r, u(r), v(r))}{r} d r\right) d s\right\} \\
& \leq \frac{1}{\left|A_{1}\right|}\left(\int_{1}^{t} s^{k-1}(\log s)^{q-2} d s\right)\left[\sum_{i=1}^{m} \frac{\left|\lambda_{i}\right|}{\Gamma\left(\alpha_{i}\right) \Gamma(q-1)} \int_{1}^{\eta_{i}}\left(\log \frac{\eta_{i}}{s}\right)^{\alpha_{i}-1} s^{-k-1}\right. \\
& \times\left(\int _ { 1 } ^ { s } r ^ { k - 1 } \left(\int_{1}^{r}\left(\log \frac{r}{m}\right)^{q-2}\right.\right. \\
& \left.\left.\times \frac{(|f(m, u(m), v(m))-f(m, 0,0)|+|f(m, 0,0)|)}{m} d m\right) d r\right) d s \\
& +\sum_{j=1}^{n} \frac{\left|\mu_{j}\right|}{\Gamma\left(\beta_{j}\right) \Gamma(q-1)} \int_{1}^{\xi_{j}}\left(\log \frac{\xi_{j}}{s}\right)^{\beta_{j}-1} s^{-k-1}\left(\int _ { 1 } ^ { s } r ^ { k - 1 } \left(\int_{1}^{r}\left(\log \frac{r}{m}\right)^{q-2}\right.\right. \\
& \left.\left.\times \frac{(|f(m, u(m), v(m))-f(m, 0,0)|+|f(m, 0,0)|)}{m} d m\right) d r\right) d s \\
& +\sum_{j=1}^{n} \frac{\left|\mu_{j}\right|}{\Gamma\left(\beta_{j}\right) \Gamma(q-1)} \int_{1}^{e}\left(\log \frac{e}{s}\right)^{\beta_{j}-1} s^{-1}\left(\int _ { 1 } ^ { e } r ^ { k - 1 } \left(\int_{1}^{r}\left(\log \frac{r}{m}\right)^{q-2}\right.\right. \\
& \left.\left.\left.\times \frac{(|f(m, u(m), v(m))-f(m, 0,0)|+|f(m, 0,0)|)}{m} d m\right) d r\right) d s\right] \\
& +\frac{1}{\Gamma(q-1)} \int_{1}^{t} s^{k-1}\left(\int_{1}^{s}\left(\log \frac{s}{r}\right)^{q-2}\right. \\
& \left.\times \frac{(|f(r, u(r), v(r))-f(r, 0,0)|+|f(r, 0,0)|)}{r} d r\right) d s \\
& \leq\left(m_{1}\|u\|+m_{2}\|v\|+N_{1}\right) \\
& \times\left[\frac { 1 } { | A _ { 1 } | } \left(\sum_{i=1}^{m} \frac{\left|\lambda_{i}\right|\left(\log \eta_{i}\right)^{\alpha_{i}+q}}{(q-1) \Gamma\left(\alpha_{i}+1\right) \Gamma(q+1)}\right.\right. \\
& \left.+\sum_{j=1}^{n} \frac{\left|\mu_{j}\right|\left(\log \xi_{j}\right)^{\beta_{j}+q}}{(q-1) \Gamma\left(\beta_{j}+1\right) \Gamma(q+1)}\right) \\
& \left.+\sum_{j=1}^{n} \frac{\left|\mu_{j}\right|}{(q-1) \Gamma\left(\beta_{j}+1\right) \Gamma(q+1)}\right] \\
& =M_{1}\left[m_{1}\|u\|+m_{2}\|v\|+N_{1}\right] \\
& =M_{1}\left[\left(m_{1}+m_{2}\right) r+N_{1}\right] \text {. }
\end{aligned}
$$

Hence,

$$
\left|\mathcal{T}_{1}(u, v)(t)\right| \leq M_{1}\left[\left(m_{1}+m_{2}\right) r+N_{1}\right]
$$

In the same way, we can obtain that

$$
\left|\mathcal{T}_{2}(u, v)(t)\right| \leq M_{2}\left[\left(n_{1}+n_{2}\right) r+N_{2}\right]
$$


Consequently, $|\mathcal{T}(u, v)(t)| \leq r$. Now, for $\left(u_{1}, v_{1}\right),\left(u_{2}, v_{2}\right) \in X \times Y$, and for any $t \in[1, e]$, we get

$$
\begin{aligned}
& \left|\mathcal{T}_{1}\left(u_{2}, v_{2}\right)(t)-\mathcal{T}_{1}\left(u_{1}, v_{1}\right)(t)\right| \\
\leq & \frac{1}{\left|A_{1}\right|}\left(t^{-k} \int_{1}^{t} s^{k-1}(\log s)^{q-2} d s\right)\left[\sum_{i=1}^{m} \frac{\left|\lambda_{i}\right|}{\Gamma\left(\alpha_{i}\right) \Gamma(q-1)} \int_{1}^{\eta_{i}}\left(\log \frac{\eta_{i}}{s}\right)^{\alpha_{i}-1} s^{-k-1}\right. \\
& \times\left(\int _ { 1 } ^ { s } r ^ { k - 1 } \left(\int_{1}^{r}\left(\log \frac{r}{m}\right)^{q-2}\right.\right. \\
& \left.\left.\frac{\left|f\left(m, u_{2}(m), v_{2}(m)\right)-f\left(m, u_{1}(m), v_{1}(m)\right)\right|}{m} d m\right) d r\right) d s \\
& +\sum_{j=1}^{n} \frac{\left|\mu_{j}\right|}{\Gamma\left(\beta_{j}\right) \Gamma(q-1)} \int_{1}^{\xi_{j}}\left(\log \frac{\xi_{j}}{s}\right)^{\beta_{j}-1} s^{-k-1} \\
& \times\left(\int _ { 1 } ^ { s } r ^ { k - 1 } \left(\int_{1}^{r}\left(\log \frac{r}{m}\right)^{q-2}\right.\right. \\
& \left.\left.\frac{\left|f\left(m, u_{2}(m), v_{2}(m)\right)-f\left(m, u_{1}(m), v_{1}(m)\right)\right|}{m} d m\right) d r\right) d s \\
& \sum_{j=1}^{n} \frac{\left|\mu_{j}\right|}{\Gamma\left(\beta_{j}\right) \Gamma(q-1)} \int_{1}^{e}\left(\log \frac{e}{s}\right)^{\beta_{j}-1} s^{-1} \\
& \times\left(M _ { 1 } ^ { e } r ^ { k - 1 } \left(\int_{1}^{r}\left(\log \frac{r}{m}\right)^{q-2}\right.\right. \\
\leq & M_{1}\left(m_{1}+m_{2}\right)\left(\left\|u_{2}-u_{1}\right\|+\left\|v_{2}-v_{1}\right\|\right), \\
& +\frac{\left|f\left(r, u_{2}(r), v_{2}(r)\right)-f\left(r, u_{1}(r), v_{1}(r)\right)\right|}{\Gamma(q-1)} \int_{1}^{t} s^{k-1}\left(\int_{1}^{s}\left(\log \frac{s}{r}\right)^{q-2} d s\right. \\
& \left.\left.\left.\frac{f\left(m, u_{2}(m), v_{2}(m)\right)-f\left(m, u_{1}(m), v_{1}(m)\right) \mid}{m} d m\right) d r\right) d s\right]
\end{aligned}
$$

and consequently, we obtain

(3.6) $\left|\mathcal{T}_{1}\left(u_{2}, v_{2}\right)(t)-\mathcal{T}_{1}\left(u_{1}, v_{1}\right)(t)\right| \leq M_{1}\left(m_{1}+m_{2}\right)\left(\left\|u_{2}-u_{1}\right\|+\left\|v_{2}-v_{1}\right\|\right)$.

Similarly,

$$
\left|\mathcal{T}_{2}\left(u_{2}, v_{2}\right)(t)-\mathcal{T}_{2}\left(u_{1}, v_{1}\right)(t)\right| \leq M_{2}\left(n_{1}+n_{2}\right)\left(\left\|u_{2}-u_{1}\right\|+\left\|v_{2}-v_{1}\right\|\right) .
$$

It follows from (3.6) and (3.7) that

$$
\left|\mathcal{T}\left(u_{2}, v_{2}\right)(t)-\mathcal{T}\left(u_{1}, v_{1}\right)(t)\right| \leq\left[M_{1}\left(m_{1}+m_{2}\right)+M_{2}\left(n_{1}+n_{2}\right)\right]
$$




$$
\times\left(\left\|u_{2}-u_{1}\right\|+\left\|v_{2}-v_{1}\right\|\right) .
$$

Since $M_{1}\left(m_{1}+m_{2}\right)+M_{2}\left(n_{1}+n_{2}\right)<1$, therefore, $\mathcal{T}$ is a contraction operator. So, by Banach fixed point theorem, the operator $\mathcal{T}$ has a unique fixed point, which is the unique solution of problem (1.1). This completes the proof.

In the next result, we prove the existence of solutions for the problem (1.1) by applying Leray-Schauder alternative.

Lemma 3.1 (Leray-Schauder alternative [7]). Let $F: E \rightarrow E$ be a completely continuous operator (i.e., a map that restricted to any bounded set in $E$ is compact). Let

$$
\aleph(F)=\{x \in E \mid x=\lambda F(x) \text { for some } 0<\lambda<1\} .
$$

Then either the set $\aleph(F)$ is unbounded or $F$ has at least one fixed point.

Theorem 3.2. Assume that there exist real constants $k_{i}, \lambda_{i}>0, i=1,2$, and $k_{0}>$ $0, \lambda_{0}>0$ such that for all $x_{i} \in \mathbb{R}, i=1,2$, we have

$$
\left|f\left(t, x_{1}, x_{2}\right)\right| \leq k_{0}+k_{1}\left|x_{1}\right|+k_{2}\left|x_{2}\right|
$$

and

$$
\left|g\left(t, x_{1}, x_{2}\right)\right| \leq \lambda_{0}+\lambda_{1}\left|x_{1}\right|+\lambda_{2}\left|x_{2}\right|
$$

In addition, also assume that

$$
\left(M_{1} k_{1}+M_{2} \lambda_{1}\right) \leq 1 \quad \text { and } \quad\left(M_{1} k_{2}+M_{2} \lambda_{2}\right) \leq 1,
$$

where $M_{i}, i=1,2$, are given by (3.3) and (3.4). Then there exists at least one solution for the boundary value problem (1.1).

Proof. First we show that the operator $\mathcal{T}: X \times Y \rightarrow X \times Y$ is completely continuous. By continuity of functions $\mathrm{f}$ and $\mathrm{g}$ the operator $\mathcal{T}$ is continuous.

Let $\Theta \subset X \times Y$ be bounded. Then there exist positive constants $L_{1}$ and $L_{2}$ such that

$$
|f(t, u(t), v(t))| \leq L_{1}, \quad|g(t, u(t), v(t))| \leq L_{2}, \quad \text { for all }(u, v) \in \Theta .
$$

For any $(u, v) \in \Theta$, we have

$$
\begin{aligned}
\left\|\mathcal{T}_{1}(u, v)(t)\right\| \leq & \frac{1}{\left|A_{1}\right|}\left(\int_{1}^{t} s^{k-1}(\log s)^{q-2} d s\right)\left[\sum_{i=1}^{m} \frac{\left|\lambda_{i}\right|}{\Gamma\left(\alpha_{i}\right) \Gamma(q-1)} \int_{1}^{\eta_{i}}\left(\log \frac{\eta_{i}}{s}\right)^{\alpha_{i}-1} s^{-k-1}\right. \\
& \times\left(\int_{1}^{s} r^{k-1}\left(\int_{1}^{r}\left(\log \frac{r}{m}\right)^{q-2} \frac{|f(m, u(m), v(m))|}{m} d m\right) d r\right) d s \\
& +\sum_{j=1}^{n} \frac{\left|\mu_{j}\right|}{\Gamma\left(\beta_{j}\right) \Gamma(q-1)} \int_{1}^{\xi_{j}}\left(\log \frac{\xi_{j}}{s}\right)^{\beta_{j}-1} s^{-k-1} \\
& \times\left(\int_{1}^{s} r^{k-1}\left(\int_{1}^{r}\left(\log \frac{r}{m}\right)^{q-2} \frac{|f(m, u(m), v(m))|}{m} d m\right) d r\right) d s
\end{aligned}
$$




$$
\begin{aligned}
& +\sum_{j=1}^{n} \frac{\left|\mu_{j}\right|}{\Gamma\left(\beta_{j}\right) \Gamma(q-1)} \int_{1}^{e}\left(\log \frac{e}{s}\right)^{\beta_{j}-1} s^{-1} \\
& \left.\times\left(\int_{1}^{e} r^{k-1}\left(\int_{1}^{r}\left(\log \frac{r}{m}\right)^{q-2} \frac{(|f(m, u(m), v(m))|}{m} d m\right) d r\right) d s\right] \\
& +\frac{1}{\Gamma(q-1)} \int_{1}^{t} s^{k-1}\left(\int_{1}^{s}\left(\log \frac{s}{r}\right)^{q-2} \frac{(|f(r, u(r), v(r))|}{r} d r\right) d s \\
& \leq \frac{L_{1}}{\left|A_{1}\right|}\left(\int_{1}^{t} s^{k-1}(\log s)^{q-2} d s\right)\left[\sum_{i=1}^{m} \frac{\left|\lambda_{i}\right|}{\Gamma\left(\alpha_{i}\right) \Gamma(q-1)} \int_{1}^{\eta_{i}}\left(\log \frac{\eta_{i}}{s}\right)^{\alpha_{i}-1} s^{-k-1}\right. \\
& \times\left(\int_{1}^{s} r^{k-1}\left(\int_{1}^{r}\left(\log \frac{r}{m}\right)^{q-2} \frac{1}{m} d m\right) d r\right) d s \\
& +\sum_{j=1}^{n} \frac{\left|\mu_{j}\right|}{\Gamma\left(\beta_{j}\right) \Gamma(q-1)} \int_{1}^{\xi_{j}}\left(\log \frac{\xi_{j}}{s}\right)^{\beta_{j}-1} s^{-k-1} \\
& \times\left(\int_{1}^{s} r^{k-1}\left(\int_{1}^{r}\left(\log \frac{r}{m}\right)^{q-2} \frac{1}{m} d m\right) d r\right) d s \\
& +\sum_{j=1}^{n} \frac{\left|\mu_{j}\right|}{\Gamma\left(\beta_{j}\right) \Gamma(q-1)} \int_{1}^{e}\left(\log \frac{e}{s}\right)^{\beta_{j}-1} s^{-1} \\
& \left.\times\left(\int_{1}^{e} r^{k-1}\left(\int_{1}^{r}\left(\log \frac{r}{m}\right)^{q-2} \frac{1}{m} d m\right) d r\right) d s\right] \\
& +\frac{L_{1}}{\Gamma(q-1)} \int_{1}^{t} s^{k-1}\left(\int_{1}^{s}\left(\log \frac{s}{r}\right)^{q-2} \frac{1}{r} d r\right) d s
\end{aligned}
$$

which implies that

$$
\begin{aligned}
\left\|\mathcal{T}_{1}(u, v)(t)\right\| \leq & L_{1}\left\{\frac { 1 } { | A _ { 1 } | } \left(\sum_{i=1}^{m} \frac{\left|\lambda_{i}\right|\left(\log \eta_{i}\right)^{\alpha_{i}+q}}{(q-1) \Gamma\left(\alpha_{i}+1\right) \Gamma(q+1)}\right.\right. \\
& \left.+\sum_{j=1}^{n} \frac{\left|\mu_{j}\right|\left(\log \xi_{j}\right)^{\beta_{j}+q}}{(q-1) \Gamma\left(\beta_{j}+1\right) \Gamma(q+1)}\right) \\
& \left.+\sum_{j=1}^{n} \frac{\left|\mu_{j}\right|}{(q-1) \Gamma\left(\beta_{j}+1\right) \Gamma(q+1)}\right\} \\
= & L_{1} M_{1} .
\end{aligned}
$$

Similarly, we get

$$
\begin{aligned}
\left\|\mathcal{T}_{2}(u, v)(t)\right\| \leq & L_{2}\left\{\frac { 1 } { | A _ { 2 } | } \left(\sum_{i=1}^{m} \frac{\left|\rho_{i}\right|\left(\log \theta_{i}\right)^{\gamma_{i}+p}}{(p-1) \Gamma\left(\gamma_{i}+1\right) \Gamma(p+1)}\right.\right. \\
& \left.+\sum_{j=1}^{n} \frac{\left|\kappa_{j}\right|\left(\log \zeta_{j}\right)^{\delta_{j}+p}}{(p-1) \Gamma\left(\delta_{j}+1\right) \Gamma(p+1)}\right)
\end{aligned}
$$




$$
\begin{aligned}
& \left.+\sum_{j=1}^{n} \frac{\left|\kappa_{j}\right|}{(p-1) \Gamma\left(\delta_{j}+1\right) \Gamma(p+1)}\right\} \\
= & L_{2} M_{2} .
\end{aligned}
$$

Thus, it follows from the above inequalities that the operator $\mathcal{T}$ is uniformly bounded.

Next, we show that $\mathcal{T}$ is equicontinuous. Let $t_{1}, t_{2} \in[1, e]$ with $t_{1}<t_{2}$. Then we have

$$
\begin{aligned}
\mid & \mathcal{T}_{1}\left(u\left(t_{2}\right), v\left(t_{2}\right)\right)-\mathcal{T}_{1}\left(u\left(t_{1}\right), v\left(t_{1}\right)\right) \mid \\
\leq & \frac{L_{1}}{\left|A_{1}\right|}\left(\frac{\left|t_{1}^{k}-t_{2}^{k}\right|}{t_{1}^{k} t_{2}^{k}} \int_{1}^{t_{1}} s^{k-1}(\log s)^{q-2} d s+t_{2}^{-k} \int_{t_{1}}^{t_{2}} s^{k-1}(\log s)^{q-2} d s\right) \\
& \times\left[\sum_{i=1}^{m} \frac{\left|\lambda_{i}\right|}{\Gamma\left(\alpha_{i}\right) \Gamma(q-1)} \int_{1}^{\eta_{i}}\left(\log \frac{\eta_{i}}{s}\right)^{\alpha_{i}-1} s^{-k-1}\right. \\
& \times\left(\int_{1}^{s} r^{k-1}\left(\int_{1}^{r}\left(\log \frac{r}{m}\right)^{q-2} \frac{1}{m} d m\right) d r\right) d s \\
& +\sum_{j=1}^{n} \frac{\left|\mu_{j}\right|}{\Gamma\left(\beta_{j}\right) \Gamma(q-1)} \int_{1}^{\xi_{j}}\left(\log \frac{\xi_{j}}{s}\right)^{\beta_{j}-1} s^{-k-1} \\
& \times\left(\int_{1}^{s} r^{k-1}\left(\int_{1}^{r}\left(\log \frac{r}{m}\right)^{q-2} \frac{1}{m} d m\right) d r\right) d s \\
& +\sum_{j=1}^{n} \frac{\left|\mu_{j}\right|}{\Gamma\left(\beta_{j}\right) \Gamma(q-1)} \int_{1}^{e}\left(\log \frac{e}{s}\right)^{\beta_{j}-1} s^{-1} \\
& \left.\times\left(\int_{1}^{e} r^{k-1}\left(\int_{1}^{r}\left(\log \frac{r}{m}\right)^{q-2} \frac{1}{m} d m\right) d r\right) d s\right] \\
& +\frac{L_{1}}{\Gamma(q-1)}\left[\frac{\left|t_{1}^{k}-t_{2}^{k}\right|}{t_{1}^{k} t_{2}^{k}} \int_{1}^{t_{1}} s^{k-1}\left(\int_{1}^{s}\left(\log \frac{s}{r}\right)^{q-2} \frac{1}{r} d r\right) d s\right. \\
& \left.+t_{2}^{-k} \int_{t_{1}}^{t_{2}} s^{k-1}\left(\int_{1}^{s}\left(\log \frac{s}{r}\right)^{q-2} \frac{1}{r} d r\right) d s\right],
\end{aligned}
$$

which implies that

$$
\left|\mathcal{T}_{1}\left(u\left(t_{2}\right), v\left(t_{2}\right)\right)-\mathcal{T}_{1}\left(u\left(t_{1}\right), v\left(t_{1}\right)\right)\right| \rightarrow 0 \quad \text { as } \quad t_{2}-t_{1} \rightarrow 0
$$

Analogously, we can obtain

$$
\left|\mathcal{T}_{2}\left(u\left(t_{2}\right), v\left(t_{2}\right)\right)-\mathcal{T}_{2}\left(u\left(t_{1}\right), v\left(t_{1}\right)\right)\right| \rightarrow 0 \quad \text { as } \quad t_{2}-t_{1} \rightarrow 0
$$

From (3.9) and (3.10), it is obvious that $\mathcal{T}$ is equicontinuous and thus is completely continuous.

Finally, it will be verified that the set $\aleph=\{(u, v) \in X \times Y:(u, v)=\lambda \mathcal{T}(u, v), 0 \leq$ $\lambda \leq 1\}$ is bounded. Let $(u, v) \in \aleph$, then $(u, v)=\lambda \mathcal{T}(u, v)$. For any $t \in[1, e]$, we have

$$
u(t)=\lambda \mathcal{T}_{1}(u, v)(t), \quad v(t)=\lambda \mathcal{T}_{2}(u, v)(t) .
$$


Then

$$
\begin{aligned}
|u(t)| \leq & \left(k_{0}+k_{1}\|u\|+k_{2}\|v\|\right)\left[\frac { 1 } { | A _ { 1 } | } \left(\sum_{i=1}^{m} \frac{\left|\lambda_{i}\right|\left(\log \eta_{i}\right)^{\alpha_{i}+q}}{(q-1) \Gamma\left(\alpha_{i}+1\right) \Gamma(q+1)}\right.\right. \\
& \left.\left.+\sum_{j=1}^{n} \frac{\left|\mu_{j}\right|\left(\log \xi_{j}\right)^{\beta_{j}+q}}{(q-1) \Gamma\left(\beta_{j}+1\right) \Gamma(q+1)}\right)+\sum_{j=1}^{n} \frac{\left|\mu_{j}\right|}{(q-1) \Gamma\left(\beta_{j}+1\right) \Gamma(q+1)}\right]
\end{aligned}
$$

and

$$
\begin{aligned}
|v(t)| \leq & \left(\lambda_{0}+\lambda_{1}\|u\|+\lambda_{2}\|v\|\right)\left[\frac { 1 } { | A _ { 2 } | } \left(\sum_{i=1}^{m} \frac{\left|\rho_{i}\right|\left(\log \theta_{i}\right)^{\gamma_{i}+p}}{(p-1) \Gamma\left(\gamma_{i}+1\right) \Gamma(p+1)}\right.\right. \\
& \left.\left.+\sum_{j=1}^{n} \frac{\left|\kappa_{j}\right|\left(\log \zeta_{j}\right)^{\delta_{j}+p}}{(p-1) \Gamma\left(\delta_{j}+1\right) \Gamma(p+1)}\right)+\sum_{j=1}^{n} \frac{\left|\kappa_{j}\right|}{(p-1) \Gamma\left(\delta_{j}+1\right) \Gamma(p+1)}\right] .
\end{aligned}
$$

Hence, we have

$$
\|u(t)\| \leq\left(k_{0}+k_{1}\|u\|+k_{2}\|v\|\right) M_{1}
$$

and

$$
\|v(t)\| \leq\left(\lambda_{0}+\lambda_{1}\|u\|+\lambda_{2}\|v\|\right) M_{2}
$$

which implies that

$$
\|u(t)\|+\|v(t)\| \leq\left(M_{1} k_{0}+M_{2} \lambda_{0}\right)+\left(M_{1} k_{1}+M_{2} \lambda_{1}\right)\|u\|+\left(M_{1} k_{2}+M_{2} \lambda_{2}\right)\|v\| .
$$

Consequently,

$$
\|(u, v)\| \leq \frac{M_{1} k_{0}+M_{2} \lambda_{0}}{M_{0}},
$$

for any $t \in[1, e]$, where $M_{0}$ is defined by (3.5), which proves that $\aleph$ is bounded. Thus, by Lemma 3.1 the operator $\mathcal{T}$ has at least one fixed point. Hence, the boundary value problem (1.1) has at least one solution. The proof is complete.

\section{Hyers-Ulam Stability of System (1.1)}

This section is devoted to the investigation of the Hyers-Ulam stability of our proposed system. Let $\varepsilon_{1}, \varepsilon_{2}>0$ such that:

$$
\left\{\begin{array}{l}
\left|\left(D^{q}+k D^{q-1}\right) u(t)-f(t, u(t), v(t))\right| \leq \varepsilon_{1}, \quad t \in[1, e] \\
\left|\left(D^{p}+k D^{p-1}\right) v(t)-g(t, u(t), v(t))\right| \leq \varepsilon_{2}, \quad t \in[1, e]
\end{array}\right.
$$

Definition 4.1. Problem (1.1) is said to be Hyers-Ulam stable if there exist $M_{i}>0$, $i=1,2$, such that, for given $\varepsilon_{1}, \varepsilon_{2}>0$ and for each solution $(u, v) \in C\left([1, e] \times \mathbb{R}^{\sharp}, \mathbb{R}\right)$ of inequality (4.1), there exists a solution $\left(u^{*}, v^{*}\right) \in C\left([1, e] \times \mathbb{R}^{\not}, \mathbb{R}\right)$ of problem (1.1) with

$$
\left\{\begin{array}{l}
\left|u(t)-u^{*}(t)\right| \leq M_{1} \varepsilon_{1}, \quad t \in[1, e] \\
\left|u(t)-v^{*}(t)\right| \leq M_{2} \varepsilon_{2}, \quad t \in[1, e] .
\end{array}\right.
$$

Remark 4.1. A $(u, v)$ is a solution of inequality (4.1) if there exist functions $Q_{i} \in$ $C([1, e], \mathbb{R}), i=1,2$ which depend on $u, v$ respectively such that 
- $\left|Q_{1}(t)\right| \leq \varepsilon_{1},\left|Q_{2}(t)\right| \leq \varepsilon_{2}, t \in[1, e]$, and

$$
\begin{cases}\left(D^{q}+k D^{q-1}\right) u(t)=f(t, u(t), v(t))+Q_{1}(t), & t \in[1, e], \\ \left(D^{p}+k D^{p-1}\right) v(t)=g(t, u(t), v(t))+Q_{2}(t), & t \in[1, e] .\end{cases}
$$

Remark 4.2. If $(x, y)$ represents a solution of inequality $(4.1)$, then $(x, y)$ is a solution of following inequality:

$$
\left\{\begin{array}{l}
\left|x(t)-x^{*}(t)\right| \leq M_{1} \varepsilon_{1}, \quad t \in[1, e] \\
\left|y(t)-y^{*}(t)\right| \leq M_{2} \varepsilon_{2}, \quad t \in[1, e] .
\end{array}\right.
$$

As from Remark 4.1, we have

$$
\begin{cases}\left(D^{q}+k D^{q-1}\right) u(t)=f(t, u(t), v(t))+Q_{1}(t), & t \in[1, e] \\ \left(D^{p}+k D^{p-1}\right) v(t)=g(t, u(t), v(t))+Q_{2}(t), & t \in[1, e] .\end{cases}
$$

With the help of Definition 4.1 and Remark 4.1, we verified Remark 4.2, in the following lines

$$
\begin{aligned}
\mid u(t)-\frac{1}{A_{1}}\left(t^{-k} \int_{1}^{t} s^{k-1}(\log s)^{q-2} d s\right)\left[\sum_{i=1}^{m} \frac{\lambda_{i}}{\Gamma\left(\alpha_{i}\right) \Gamma(q-1)} \int_{1}^{\eta_{i}}\left(\log \frac{\eta_{i}}{s}\right)^{\alpha_{i}-1} s^{-k-1}\right. \\
\times\left(\int_{1}^{s} r^{k-1}\left(\int_{1}^{r}\left(\log \frac{r}{m}\right)^{q-2} \frac{f(m, u(m), v(m))}{m} d m\right) d r\right) d s \\
+\sum_{j=1}^{n} \frac{\mu_{j}}{\Gamma\left(\beta_{j}\right) \Gamma(q-1)} \int_{1}^{\xi_{j}}\left(\log \frac{\xi_{j}}{s}\right)^{\beta_{j}-1} s^{-k-1} \\
\times\left(\int_{1}^{s} r^{k-1}\left(\int_{1}^{r}\left(\log \frac{r}{m}\right)^{q-2} \frac{f(m, u(m), v(m))}{m} d m\right) d r\right) d s \\
-\sum_{j=1}^{n} \frac{e^{-k} \mu_{j}}{\Gamma\left(\beta_{j}\right) \Gamma(q-1)} \int_{1}^{e}\left(\log \frac{e}{s}\right)^{\beta_{j}-1} s^{-1} \\
\left.\quad \times\left(\int_{1}^{e} r^{k-1}\left(\int_{1}^{r}\left(\log \frac{r}{m}\right)^{q-2} \frac{f(m, u(m), v(m))}{m} d m\right) d r\right) d s\right] \\
-\frac{t^{-k}}{\Gamma(q-1)} \int_{1}^{t} s^{k-1}\left(\int_{1}^{s}\left(\log \frac{s}{r}\right)^{q-2} \frac{f(r, u(r), v(r))}{r} d r\right) d s \mid \\
=\mid \frac{1}{A_{1}}\left(t^{-k} \int_{1}^{t} s^{k-1}(\log s)^{q-2} d s\right)\left[\sum_{i=1}^{m} \frac{\lambda_{i}}{\Gamma\left(\alpha_{i}\right) \Gamma(q-1)} \int_{1}^{\eta_{i}}\left(\log \frac{\eta_{i}}{s}\right)^{\alpha_{i}-1} s^{-k-1}\right. \\
\quad \times\left(\int_{1}^{s} r^{k-1}\left(\int_{1}^{r}\left(\log \frac{r}{m}\right)^{q-2} \frac{\left[f(m, u(m), v(m))+Q_{1}(m)\right]}{m} d m\right) d r\right) d s \\
\quad+\sum_{j=1}^{n} \frac{\mu_{j}}{\Gamma\left(\beta_{j}\right) \Gamma(q-1)} \int_{1}^{\xi_{j}}\left(\log \frac{\xi_{j}}{s}\right)^{\beta_{j}-1} s^{-k-1}
\end{aligned}
$$




$$
\begin{aligned}
& \times\left(\int_{1}^{s} r^{k-1}\left(\int_{1}^{r}\left(\log \frac{r}{m}\right)^{q-2} \frac{\left[f(m, u(m), v(m))+Q_{1}(m)\right]}{m} d m\right) d r\right) d s \\
& -\sum_{j=1}^{n} \frac{e^{-k} \mu_{j}}{\Gamma\left(\beta_{j}\right) \Gamma(q-1)} \int_{1}^{e}\left(\log \frac{e}{s}\right)^{\beta_{j}-1} s^{-1} \\
& \left.\times\left(\int_{1}^{e} r^{k-1}\left(\int_{1}^{r}\left(\log \frac{r}{m}\right)^{q-2} \frac{\left[f(m, u(m), v(m))+Q_{1}(m)\right]}{m} d m\right) d r\right) d s\right] \\
& +\frac{t^{-k}}{\Gamma(q-1)} \int_{1}^{t} s^{k-1}\left(\int_{1}^{s}\left(\log \frac{s}{r}\right)^{q-2} \frac{\left[f(r, u(r), v(r))+Q_{1}(r)\right]}{r} d r\right) d s \\
& -\frac{1}{A_{1}}\left(t^{-k} \int_{1}^{t} s^{k-1}(\log s)^{q-2} d s\right)\left[\sum_{i=1}^{m} \frac{\lambda_{i}}{\Gamma\left(\alpha_{i}\right) \Gamma(q-1)} \int_{1}^{\eta_{i}}\left(\log \frac{\eta_{i}}{s}\right)^{\alpha_{i}-1} s^{-k-1}\right. \\
& \times\left(\int_{1}^{s} r^{k-1}\left(\int_{1}^{r}\left(\log \frac{r}{m}\right)^{q-2} \frac{f(m, u(m), v(m))}{m} d m\right) d r\right) d s \\
& +\sum_{j=1}^{n} \frac{\mu_{j}}{\Gamma\left(\beta_{j}\right) \Gamma(q-1)} \int_{1}^{\xi_{j}}\left(\log \frac{\xi_{j}}{s}\right)^{\beta_{j}-1} s^{-k-1} \\
& \times\left(\int_{1}^{s} r^{k-1}\left(\int_{1}^{r}\left(\log \frac{r}{m}\right)^{q-2} \frac{f(m, u(m), v(m))}{m} d m\right) d r\right) d s \\
& -\sum_{j=1}^{n} \frac{e^{-k} \mu_{j}}{\Gamma\left(\beta_{j}\right) \Gamma(q-1)} \int_{1}^{e}\left(\log \frac{e}{s}\right)^{\beta_{j}-1} s^{-1} \\
& \left.\times\left(\int_{1}^{e} r^{k-1}\left(\int_{1}^{r}\left(\log \frac{r}{m}\right)^{q-2} \frac{f(m, u(m), v(m))}{m} d m\right) d r\right) d s\right] \\
& -\frac{t^{-k}}{\Gamma(q-1)} \int_{1}^{t} s^{k-1}\left(\int_{1}^{s}\left(\log \frac{s}{r}\right)^{q-2} \frac{f(r, u(r), v(r))}{r} d r\right) d s \mid \\
& \leq \varepsilon_{1}\left[\frac{1}{\left|A_{1}\right|}\left(\sum_{i=1}^{m} \frac{\left|\lambda_{i}\right|\left(\log \eta_{i}\right)^{\alpha_{i}+q}}{(q-1) \Gamma\left(\alpha_{i}+1\right) \Gamma(q+1)}+\sum_{j=1}^{n} \frac{\left|\mu_{j}\right|\left(\log \xi_{j}\right)^{\beta_{j}+q}}{(q-1) \Gamma\left(\beta_{j}+1\right) \Gamma(q+1)}\right)\right. \\
& \left.+\sum_{j=1}^{n} \frac{\left|\mu_{j}\right|}{(q-1) \Gamma\left(\beta_{j}+1\right) \Gamma(q+1)}\right] \\
& +\varepsilon_{2}\left[\frac{1}{\left|A_{2}\right|}\left(\sum_{i=1}^{m} \frac{\left|\rho_{i}\right|\left(\log \theta_{i}\right)^{\gamma_{i}+p}}{(p-1) \Gamma\left(\gamma_{i}+1\right) \Gamma(p+1)}+\sum_{j=1}^{n} \frac{\left|\kappa_{j}\right|\left(\log \zeta_{j}\right)^{\delta_{j}+p}}{(p-1) \Gamma\left(\delta_{j}+1\right) \Gamma(p+1)}\right)\right. \\
& \left.+\sum_{j=1}^{n} \frac{\left|\kappa_{j}\right|}{(p-1) \Gamma\left(\delta_{j}+1\right) \Gamma(p+1)}\right] \\
& =M_{1} \varepsilon_{1} \text {. }
\end{aligned}
$$


By the same method, we can obtain that

$$
\left|y(t)-y^{*}(t)\right| \leq M_{2} \varepsilon_{2},
$$

where $M_{i}, i=1,2$, are given by (3.3) and (3.4). Hence, Remark 4.2 is verified, with the help of (4.4) and (4.5). Thus the nonlinear sequential coupled system of Hadamard fractional differential equations is Hyers-Ulam stable and consequently, the system (1.1) is Hyers-Ulam stable.

Example 4.1. Consider the following coupled system of Hadamard fractional differential equation:

$$
\left\{\begin{array}{l}
\left(D^{3 / 2}+3 D^{1 / 2}\right) u(t)=\frac{|u(t)|}{(t+3)^{4}+(1+|u(t)|)}+\frac{1}{27(1+|v(t)|)}+\frac{1}{81}, \quad t \in[1, e], \\
\left(D^{3 / 2}+3 D^{1 / 2}\right) v(t)=\frac{\sin (2 \pi|u(t)|)}{40 \pi}+\frac{1}{10 \sqrt{t+4}}+\frac{|v(t)|}{60(1+|v(t)|)}, \quad t \in[1, e], \\
u(1)=0, \\
2 I^{1 / 4} u(5 / 4)+\frac{1}{5} I^{3 / 2} u(9 / 5)+3 I^{1 / 2} u(15 / 7) \\
=I^{2 / 3} u(e)-I^{2 / 3} u(10 / 7)+5\left(I^{9 / 7} u(e)-I^{9 / 7} u(2)\right)-2\left(I^{11 / 4} u(e)-I^{11 / 4} u(9 / 4)\right), \\
v(1)=0, \\
\frac{1}{4} I^{7 / 6} v(7 / 3)-\frac{2}{3} I^{1 / 2} v(7 / 5)-2 I^{5 / 2} v(2) \\
=4\left(I^{5} v(e)-I^{5} v(11 / 5)\right)+\frac{11}{4}\left(I^{3 / 4} v(e)-I^{3 / 4} v(13 / 16)\right)-\frac{1}{2}\left(I^{7 / 4} v(e)\right. \\
\left.-I^{7 / 4} v(1 / 3)\right) .
\end{array}\right.
$$

Here, $q=p=3 / 2, n=3, m=3, k=3, \lambda_{1}=2, \lambda_{2}=1 / 5, \lambda_{3}=3, \alpha_{1}=1 / 4$, $\alpha_{2}=3 / 2, \alpha_{3}=1 / 2, \eta_{1}=5 / 4, \eta_{2}=9 / 5, \eta_{3}=15 / 7, \mu_{1}=1, \mu_{2}=5, \mu_{3}=-2$, $\beta_{1}=2 / 3, \beta_{2}=9 / 7, \beta_{3}=11 / 4, \xi_{1}=10 / 7, \xi_{2}=2, \xi_{3}=9 / 4, \rho_{1}=1 / 4, \rho_{2}=-2 / 3$, $\rho_{3}=-2, \gamma_{1}=7 / 6, \gamma_{2}=1 / 2, \gamma_{3}=5 / 2, \theta_{1}=7 / 3, \theta_{2}=7 / 5, \theta_{3}=2, \kappa_{1}=4, \kappa_{2}=11 / 4$, $\kappa_{3}=-1 / 2, \delta_{1}=5, \delta_{2}=3 / 4, \delta_{3}=7 / 4, \zeta_{1}=11 / 5, \zeta_{2}=13 / 16, \zeta_{3}=1 / 3$. Thus,

$$
f(t, u, v)=(u(t)) /\left((t+3)^{4}+(1+|u(t)|)\right)+1 /(27(1+|v(t)|))+1 / 81
$$

and

$$
g(t, u, v)=(\sin (2 \pi|u(t)|)) / 40 \pi+1 /(10 \sqrt{t+4})+(|v(t)|) /(60(1+|v(t)|)),
$$

which implies

$$
\left|f\left(t, u_{2}, v_{2}\right)-f\left(t, u_{1}, v_{1}\right)\right| \leq(1 / 81)\left|u_{2}-u_{1}\right|+(1 / 27)\left|v_{2}-v_{1}\right|
$$

and

$$
\left|g\left(t, u_{2}, v_{2}\right)-g\left(t, u_{1}, v_{1}\right)\right| \leq(1 / 20)\left|u_{2}-u_{1}\right|+(1 / 60)\left|v_{2}-v_{1}\right| .
$$

Clearly, $m_{1}=1 / 81, m_{2}=1 / 27, n_{1}=1 / 20, n_{2}=1 / 60, M_{1} \simeq 5.695, M_{2} \simeq 6.785$, and

$$
\left[M_{1}\left(m_{1}+m_{2}\right)+M_{2}\left(n_{1}+n_{2}\right)\right] \simeq 0.732<1 .
$$


Thus, all the conditions of Theorem 3.1 are satisfied. Therefore, by Theorem 3.1, the problem (4.8) has a unique solution on $[1, e]$. Further, it is also straightforward to prove that the problem (4.8) is Hyers-Ullam stable.

\section{Conclusion}

We have discussed the existence and Hyers-Ulam stability for a sequential coupled system consisting of fractional differential equations of Hadamard type, subjected to nonlocal Hadamard fractional integral boundary conditions. The existence and uniqueness of solutions rely on Banach's contraction principle, while the existence of solutions is established by applying Leray-Schauder's alternative. As an application, an example is presented to illustrate the main results.

\section{REFERENCES}

[1] G. Adomian and G. E. Adomian, Cellular systems and aging models, Comput. Math. Appl. 11 (1985), 283-291.

[2] B. Ahmad, J. Juan, J. Nieto and A. Alseadi, A coupled system of Caputo-type sequential fractional differential equations with coupled (periodic/anti-periodic type) boundary conditions, Mediterr. J. Math. 2017 (2017), 227.

[3] B. Ahmad and S. K. Ntouayas, A fully Hadamard type integral boundary value problome of a coupled system of fractional differential equations, Fract. Calc. Appl. Anal. 17 (2014), 348-360.

[4] Z. Ali, A. Zada and K. Shah, On Ulam's stability for a coupled systems of nonlinear implicit fractional differential equations, Bull. Malays. Math. Sci. Soc. 42 (2019), 2681-2699.

[5] R. L. Bagley and P. J. Torvik, A theoretical basis for the application of fractional calculus to viscoelasticity, Journal of Rheology 27 (1983), 201-210.

[6] K. W. Blayneh, Analysis of age structured host-parasitoid model, Far East Journal of Dynamical Systems 4 (2002), 125-145.

[7] A. Granas and J. Dugundji, Fixed Point Theory, Springer-Verlag, New York, 2013.

[8] J. Hadamard, Essai sur l'etude des fonctions donnes par leur developpment de taylor, Journal des Mathématiques Pures et Appliquées 8 (1892), 86-101.

[9] R. Hilfer, Applications of Fractional Calculus in Physics, World Scientific, SIngapore, 2000.

[10] D. H. Hyers, On the stability of the linear functional equation, Proc. Natl. Acad. Sci. USA 27 (1941), 222-224.

[11] S. Karthikeyan, C. Ravichandran and T. Gunasekar, Existence results for Hadamard type fractional functional integro-differential equations with integral boundary conditions, International Journal of Engineering Research 10 (2015), 6919-6932.

[12] H. Khan, Y. Li, W. Chen, D. Baleanu and A. Khan, Existence theorems and Hyers-Ulam stability for a coupled system of fractional differential equations with p-Laplacian operator, Bound. Value Probl. 2017.

[13] H. Khan, Y. Li, H. Hongguang and A. Khan, Existence of solution and Hyers-Ulam stability for a coupled system of fractional differential equations with p-laplacian operator, J. Nonlinear Sci. Appl. 10 (2017), 5219-5229.

[14] A. A. Kilbas, H. M. Srivastava and J. J. Trujillo, Theory and Applications of Fractional Differential Equations, North-Holland Mathematics Studies, Amsterdam, 2016.

[15] D. Matignon, Stability results for fractional differential equations with applications to control processing, Computational Engineering in System Application 2 (1996), 963-968. 
[16] T. Phollakrit, S. K. Ntouyas and T. Jessada, Existence and uniqueness results for Hadamardtype fractional differential equations with nonlocal fractional integral boundary conditions, Abstr. Appl. Anal. 5 (2014), 1-9.

[17] I. Podlubny, Fractional Differential Equations, Academic Press, San Diego, 1999.

[18] J. Sabatier, O. P. Agrawal and J. A. T. Machado, Advances in Fractional Calculus, Theoretical Developments and Applications in Physics and Engineering, Springer, Dordrecht, 2007.

[19] S. G. Samko, A. A. Kilbas and O. l. Marichev, Fractional Integrals and Derivatives: Theory and Applications, Gordon and Breach, Yverdon, 1993.

[20] K. Shah and R. A. Khan, Existence and uniqueness of positive solutions to a coupled system of nonlinear fractional order differential equations with anti periodic boundary conditions, Differ. Equ. Appl. 7(2) (2015), 245-262.

[21] R. Shah and A. Zada, A fixed point approach to the stability of a nonlinear Volterra integrodiferential equation with delay, Hacet. J. Math. Stat. 47 (2018), 615-623.

[22] S. O. Shah and A. Zada, Existence, uniqueness and stability of solution to mixed integral dynamic systems with instantaneous and noninstantaneous impulses on time scales, Appl. Math. Comput. 359 (2019), 202-213.

[23] S. O. Shah, A. Zada and A. E. Hamza, Stability analysis of the first order non-linear impulsive time varying delay dynamic system on time scales, Qual. Theory Dyn. Syst. (2019), DOI $10.1007 / \mathrm{s} 12346-019-00315-\mathrm{x}$.

[24] S. Tang, A. Zada, S. Faisal, M. M. A. El-Sheikh and T. Li, Stability of higher-order nonlinear impulsive differential equations, J. Nonlinear Sci. Appl. 9 (2016), 4713-4721.

[25] J. Tariboon and W. Sudsutad, Coupled systems of Riemann-Liouville fractional differential equations with Hadamard fractional integral boundary conditions, J. Nonlinear Sci. Appl. 9 (2016), 295-308.

[26] S. M. Ulam, Problems in Modern Mathematics, John Wiley and Sons, New York, USA, 1940.

[27] J. Wang, L. Lv and Y. Zhou, Ulam stability and data dependence for fractional differential equations with Caputo derivative, Electron J. Qual. Theo. Diff. Equns. 63 (2011), 1-10.

[28] J. Wang, A. Zada and W. Ali, Ulam's-type stability of first-order impulsive differential equations with variable delay in quasi-Banach spaces, Numer. Funct. Anal. Optim. 33 (2012), 216-238.

[29] J. Wang, A. Zada and W. Ali, Ulam's-type stability of first-order impulsive differential equations with variable delay in quasi-Banach spaces, Int. J. Nonlinear Sci. 19 (2018), 553-560.

[30] A. Zada and S. Ali, Stability analysis of multi-point boundary value problem for sequential fractional differential equations with non-instantaneous impulses, Int. J. Nonlinear Sci. Numer. Simul. 19 (2018), 763-774.

[31] A. Zada, S. Ali and Y. Li, Ulam-type stability for a class of implicit fractional differential equations with non-instantaneous integral impulses and boundary condition, Adv. Difference Equ. 2017.

[32] A. Zada, W. Ali and S. Farina, Hyers-Ulam stability of nonlinear differential equations with fractional integrable impulses, Math. Methods Appl. Sci. 40 (2017), 5502-5514.

[33] A. Zada, W. Ali and C. Park, Ulam's type stability of higher order nonlinear delay differential equations via integral inequality of Grönwall-Bellman-Bihari's type, Appl. Math. Comput. 350 (2019), 60-65.

[34] A. Zada, U. Riaz and F. U. Khan, Hyers-Ulam stability of impulsive integral equations, Boll. Unione Mat. Ital. 12 (2019), 453-467.

[35] A. Zada and S. O. Shah, Hyers-Ulam stability of first-order non-linear delay differential equations with fractional integrable impulses, Hacet. J. Math. Stat. 47 (2018), 1196-1205.

[36] A. Zada, S. O. Shah and R. Shah, Hyers-Ulam stability of non-autonomous systems in terms of boundedness of Cauchy problem, Appl. Math. Comput. 271 (2015), 512-518.

[37] A. Zada, S. Shaleena and T. Li, Stability analysis of higher order nonlinear differential equations in $\beta$-normed spaces, Math. Methods Appl. Sci. 42 (2019), 1151-1166. 
[38] A. Zada, P. Wang, D. Lassoued and T. Li, Connections between Hyers-Ulam stability and uniform exponential stability of 2-periodic linear nonautonomous systems, Adv. Difference Equ. 2017.

[39] A. Zada, M. Yar and T. Li, Existence and stability analysis of nonlinearsequential coupled system of Caputo fractional differential equations with integral boundary conditions, Ann. Univ. Paedagog. Crac. Stud. Math. 17 (2018), 103-125.

${ }^{1}$ Department of MAThematics, University of PeshaWAR, Peshawar 25000, PAKISTAN

Email address: zadababo@yahoo.com, akbarzada@uop.edu.pk

Email address: mohammadyar2030@gmail.com 